\title{
The Quebec Association of Gastroenterology position paper on colorectal cancer screening - 2003
}

\author{
AN Barkun MD MSc${ }^{1}$, G Jobin $M D^{2}$, G Cousineau $M D^{3}$, S Dubé $M D^{2}, \mathrm{R}$ Lahaie $M D^{4}$, \\ $P$ Paré $M D^{5}$, B Stein $M D^{1}, R$ Wassef $M D^{4}$
}

\begin{abstract}
AN Barkun, G Jobin, G Cousineau, et al. The Quebec Association of Gastroenterology position paper on colorectal cancer screening - 2003. Can J Gastroenterol 2004;18(8):509-519.

Colorectal cancer is a leading cause of death and the third most common cancer in Canada. Evidence suggests that screening can reduce mortality rates and the cost effectiveness of a program compares favourably with initiatives for breast and cervical cancer.

The objectives of the Association des gastro-entérologues du Québec Task Force were to determine the need for a policy on screening for colorectal cancer in Quebec, to evaluate the testing methods available and to propose one or more of these alternatives as part of a formal screening program, if indicated.

Fecal occult blood testing (FOBT), endoscopy (including sigmoidoscopy and colonoscopy), barium enema and virtual colonoscopy were considered. Although most clinical efficacy data are available for FOBT and sigmoidoscopy, there are limitations to programs based on these strategies. FOBT has a high false positive rate and a low detection yield, and even a combination of these strategies will miss $24 \%$ of cancers. Colonoscopy is the best strategy to both detect and remove polyps and to diagnose colorectal cancer, with double contrast barium enema also being a sensitive detection method.

The Task Force recommended the establishment, in Quebec, of a screening program with five- to 10 -yearly double contrast barium enema or 10-yearly colonoscopy for individuals aged 50 years or older at low risk. The program should include outcome monitoring, public and professional education to increase awareness and promote compliance, and central coordination with other provincial programs. The program should be evaluated; specific billing codes for screening for colorectal cancer would help facilitate this. Formal feasibility, effectiveness and cost-effectiveness studies in Quebec are now warranted.
\end{abstract}

Key Words: Colonoscopy; Colorectal cancer; Fecal occult blood test; Guidelines; Screening

\section{RECOMMENDATIONS}

Summary based on existing evidence, and pertinent comments on screening methods

1. There exists Level I evidence that screening reduces the mortality rates from colorectal cancer (CRC)

(A recommendation). Furthermore, most cost-

effectiveness data suggest that such a program compares favourably with existing screening initiatives such as for breast and cervical cancer.

\section{Le document de principes de l'Association des gastro-entérologues du Québec sur le dépistage du cancer colorectal 2003}

Le cancer colorectal est une cause importante de décès et le troisième cancer en importance au Canada. Selon des données probantes, le dépistage peut réduire les taux de mortalité, et le rapport coût-efficacité d'un programme se compare favorablement avec les initiatives relatives au cancer du sein et du col de l'utérus.

Les objectifs du groupe de travail de l'Association des gastro-entérologues du Québec consistaient à déterminer le besoin d'une politique de dépistage du cancer colorectal au Québec, à évaluer les méthodes d'essai disponibles et à proposer une ou plusieurs de ces possibilités dans le cadre d'un programme de dépistage officiel, s'il y a lieu.

Le test de sang occulte fécal (TSOF) et l'endoscopie (y compris une sigmoïdoscopie et une coloscopie), le lavement baryté et la coloscopie virtuelle ont été envisagés. Bien que la plupart des données d'efficacité clinique soient disponibles pour le TSOF, la sigmoïdoscopie, les programmes fondés sur ces stratégies comportent des limites. Le TSOF s'associe à un taux faux positif élevé et à un faible rendement de détection, et même une combinaison de ces stratégies passera à côté de $24 \%$ des cancers. Toutefois, la coloscopie représente la meilleure stratégie à la fois pour déceler et extraire les polypes et pour diagnostiquer le cancer colorectal, et le lavement baryté en double contraste constitue une méthode de détection sensible.

Le groupe de travail a recommandé la mise en œuvre, au Québec, d'un programme de dépistage comportant un lavement baryté en double contraste aux cinq à dix ans ou une coloscopie aux dix ans pour les personnes de 50 ans ou plus à faible risque. Ce programme devrait inclure l'évaluation des issues, l'éducation publique et la formation professionnelle pour accroître la sensibilisation et promouvoir le respect du traitement et une coordination centralisée avec d'autres programmes provinciaux. Le programme devrait être évalué. Des codes de facturation précis pour le dépistage du cancer colorectal faciliteraient cette évaluation. Maintenant, des études officielles de faisabilité, d'efficacité et de rapport coût-efficacité au Québec s'imposent.

${ }^{1}$ MUHC-McGill University and the McGill University Health Centre, Montreal General Hospital site; ${ }^{2}$ Université de Montréal, Hôpital Maisonneuve-Rosemont, Centre Universitaire Affilié; ${ }^{3}$ Université de Montréal, Centre Hospitalier Universitaire de Montréal, pavillon Hôpital Notre-Dame; ${ }^{4}$ Université de Montréal, Centre Hospitalier Universitaire de Montréal, pavillon Hôpital St-Luc; ${ }^{5}$ Centre Hospitalier Affilié Universitaire de Québec, Hôpital du Saint-Sacrement, Montréal, Québec

Correspondence and reprints: Dr Gilles Jobin, L'association des gastro-entérologues du Québec, 2, Complexe Desjardins, CP 216, succursale Desjardins, Montreal, Quebec H5B 1G8. Telephone 514-350-5112, fax 514-350-5152, e-mail gjobin@videotron.ca

Received for publication February 26, 2004. Accepted April 21, 2004 


\section{TABLE 1}

Recommendations for a screening program for colorectal cancer (CRC)

1. Establish a screening program for CRC for patients aged 50 years or older who are at low risk and asymptomatic

2. Perform five- to 10 -yearly DCBE or 10 -yearly colonoscopy

3. Monitor appropriate outcomes

4. Monitor patients undergoing other screening methods (eg, FOBT and/or flexible sigmoidoscopy)

5. Include public and professional education to increase awareness and promote compliance, and central coordination of provincial programs with centralized data collection to allow ongoing evaluation of the program

6. Adopt specific billing codes for screening for $\mathrm{CRC}$ to assist in tracking the results of the program

7. Undertake immediate formal studies on feasibility, effectiveness and cost-effectiveness modelling of these recommendations adapted to the Quebec environment

8. Ensure adequate follow-up for patients in whom colonic polyps or cancer are discovered, regardless of the screening method used

DCBE Double contrast barium enema; FOBT Fecal occult blood testing

(B recommendation), a strategy that misses up to $24 \%$ of colonic lesions.

3. Colonoscopy is the best test to diagnose and remove polyps (Level II) and to diagnose CRC. Double contrast barium enema (DCBE) is also an excellent method of detection of colonic polyps and CRC (Level III). However, there exist insufficient data to assess the impact on outcomes of these strategies when used as screening methods ( $\mathrm{C}$ recommendation for both).

4. There exist insufficient test-characteristics data on other diagnostic modalities used for screening to recommend them at this time.

\section{Rationale/justification of any possible recommendations}

1. An effective screening program is likely cost effective, but there are limitations to a program based on the use of FOBT or flexible sigmoidoscopy. More specific and sensitive screening methods include DCBE and colonoscopy.

2. Issues regarding the comparative efficacy, cost and cost effectiveness of the various screening tests remain uncertain.

3. Issues of feasibility and cost effectiveness as they apply to the Quebec health care system and the population of Quebec also remain uncertain. These need to be considered to make optimal recommendations.

4. The Task Force felt that no results from ongoing or planned randomized trials in the field would be made available in the foreseeable future that would affect decisions made at this time. Therefore, recommendations for a screening program in the detection of CRC in Quebec should not be postponed pending unavailable higher quality data with regard to using colonoscopy or barium enema.

\section{Recommendations of the committee}

Based on the existing evidence from high-quality and outcome studies, and on recognized diagnostic test characteristics of the different screening tools available, the committee recommends (Table 1):

1. The rapid establishment of a screening program for CRC targeting patients aged 50 years or older at low risk of colonic neoplasia and otherwise asymptomatic;

2. The screening program should be based on the performance of five- to 10-yearly DCBE or 10-yearly colonoscopy without waiting for high-quality evidence for their use. The feasibility of such an approach in Quebec is addressed in Appendix 2;

3. The screening program should include monitoring of appropriate outcomes;

4. An effort should also be made to monitor patients undergoing what may be less effective methods of screening (such as FOBT and/or flexible sigmoidoscopy);

5. The screening program should include multiple components: public education to heighten public awareness and understanding, in order to promote compliance; professional education to increase physician awareness and patient compliance; and central coordination of all province-wide screening efforts, including a centralized data collection infrastructure to allow ongoing evaluation of the program. This initiative should follow the structure put in place for breast cancer screening;

6. Specific billing codes for screening for CRC should be adopted in the different subspecialties to assist in tracking the results of the program;

7. Formal studies on feasibility, effectiveness and costeffectiveness modelling adapted to the Quebec environment should be undertaken as soon as possible; and

8. Adequate follow-up should be ensured, regardless of the screening method used, as part of the program for patients in whom colonic polyps or cancer are discovered.

\section{Epidemiology}

\section{INTRODUCTION}

CRC is a leading cause of death in the Western world (1). In Canada, it is the third most common cancer, accounting for more than $12 \%$ of cases of cancer in both sexes. In 2001, there were an estimated 17,200 new cases and 6400 deaths from CRC in Canada, of which 4300 and 2100, respectively, were in Quebec (2).

It is widely accepted that survival rates are improved if the disease is treated in its early stages (1). The well-recognized polyp-to-cancer sequence provides the rationale for early intervention based on the diagnosis of colonic polyps or early cancers. Indeed, with the advances of endoscopic techniques that allow for both detection and removal of precursor lesions, $\mathrm{CRC}$ is now a preventable disease. 


\section{The polyp-cancer sequence}

It is generally accepted that there is a relationship between adenomatous colonic polyps and the subsequent development of carcinoma (3). The presence of adenomas in the colon and rectum of patients undergoing resection for cancer has been reported by Morson and Dawson (4) in one-third of patients, by Greene (5) in 13\% and by Helwig (6) in 50\%. Helwig (6) also found adenomas with carcinomatous transitions. The synchronicity of adenomas and cancer has been validated in numerous colonoscopy studies. Prager et al (7) followed 300 patients for 15 years following sigmoidoscopy-benign polypectomy and concluded that patients with colorectal polyps have a greater tendency to develop CRC than do unaffected persons. It is believed that the vast majority of CRCs arise from benign adenomatous polyps, and as the size of the polyp increases so does the incidence of carcinoma. An adenoma greater than $2 \mathrm{~cm}$ has a $46 \%$ chance of containing invasive carcinoma (8). The time for a polyp to develop and to go onto cancer is estimated to be between 10 and 15 years $(1,9)$.

\section{Risk factors}

Individuals with hereditary nonpolyposis colon cancer, and families with familial polyposis coli have been identified to be at high risk of developing CRC $(10,11)$. Specific aggressive screening guidelines exist for such patients (12). A genetic basis has been implicated in approximately $15 \%$ of all cancers, with $6 \%$ of all cases being attributed to the two hereditary conditions noted above (5\% for hereditary nonpolyposis colon cancer and $1 \%$ for familial polyposis coli) (1). Thus, these conditions represent a minority of all cases of colon cancer and consequently, this report will focus specifically on individuals at low risk for the development of CRC. Similarly, other high-risk groups, such as patients with longstanding ulcerative colitis and those with a family history of colonic polyps or colon cancer, will not be discussed in the present report.

Age is a significant risk factor, with less than $2 \%$ of all cancers occurring in patients under age 40 years. The risk of CRC in a 50-year-old individual is 18 to 20 times higher than that of a 30-year-old, and the risk doubles about every seven years thereafter (1). A recent cohort analysis of 906 consecutive persons aged 40 to 49 years would appear to confirm the low prevalence of lesions in this age range; there were only $3.5 \%$ of advanced neoplasias with no cancers, 0\% (95\% CI 0\% to $0.4 \%$ ) (13). These data form an important basis for subsequent recommendations based on specific age cut-offs.

\section{Treatment}

Surgical resection remains the standard therapy for CRC, but adjuvant therapy with radiation and chemotherapy has also been shown to improve the outcome of selected patients based on the nature and location of the tumour (14).

\section{METHODS}

The following screening methods were reviewed: FOBT, endoscopy (including sigmoidoscopy and colonoscopy), barium enema and virtual colonoscopy. To determine the clinical efficacy of these tests, a thorough narrative review of the literature until late 2002 was undertaken by members of the committee to identify studies using the highest level of evidence. A more detailed methodology for the selection of articles used to create this report is available on request. The present report does not include an exhaustive review of the available evidence, but rather the most relevant data applicable to screening in CRC $(1,15,16)$.

The levels of evidence and strength of recommendations referred to in the present report relate to the classification by the Canadian Task Force on the Periodic Health Examination (Appendix 1) (17).

\section{Screening tests}

\section{BACKGROUND}

FOBT: The most common protocol for screening of averagerisk individuals is a multiphase strategy, with FOBT initially, followed by colonoscopy or flexible sigmoidoscopy and barium enema for those with a positive test. Different types of FOBTs exist, all of which are designed to detect the presence of hemoglobin in the stools. Some detect a peroxidase-like activity of hemoglobin (the Hemoccult test, Beckman Coulter, USA), while others detect the porphyrin-like moiety of hemoglobin (HemeLab Inc, USA). Some tests require a specific diet and rehydration to optimize performance characteristics. Immunochemical tests have also been developed, including radial immunodiffusion, latex hemagglutination, enzyme-linked immunosorbent assay and monoclonal antibodies against hemoglobin. The most commonly used of these is the HemeSelect test (SmithKline Diagnostics, USA) (12).

Endoscopy (sigmoidoscopy/colonoscopy): An alternative to multiphasic screening for average-risk individuals is screening with sigmoidoscopy alone or colonoscopy. In both, an endoscope is inserted through the anus into the colon up to the cecum in the case of a colonoscopy, and only $40 \mathrm{~cm}$ to $60 \mathrm{~cm}$ up for a sigmoidoscopy or short colonoscopy. Colonoscopy requires more stringent preparation by the patient, more expertise by the endoscopist and is associated with higher complication rates (usually quoted at approximately two per 1000 compared to one per 10,000 for the flexible sigmoidoscopy) (18). For example, the complication rate in a series of 12,246 colonoscopies performed in a university setting was $0.1 \%$, with no deaths (12). Colonoscopy is arguably the best method of detecting colonic polyps and CRC (18).

Barium enema: The DCBE can detect CRCs in $85 \%$ to $90 \%$ of cases. Its reported sensitivity approximates $75 \%$ to $90 \%$ for adenomas of more than $1 \mathrm{~cm}$ and $50 \%$ to $80 \%$ for those of less than $1 \mathrm{~cm}$. It should be noted that the clinical importance of adenomas of less than $1 \mathrm{~cm}$ in CRC screening is not known. DCBE is relatively well tolerated and has a low rate of complication (one perforation or hemorrhage per 10,000 examinations [19]).

Virtual colonoscopy: Virtual colonoscopy is a new technique involving the examination of computerized images of the colon constructed from data obtained from an abdominal computed tomagraphic imaging scan following adequate preparation by the patient. Sensitivities of up to $91 \%$ for the detection of colonic lesions over $1 \mathrm{~cm}$ have been reported, but the false positive rate was almost $17 \%$ in recent studies (18). Future improvements to this technology may make it an attractive and important screening option (20).

When evaluating the efficacy of the various screening techniques, the reader should consider issues of generalizability attributable to operator expertise, as well as publication bias, which favour the reporting of optimal test performance results, particularly early on in the development of a technology. 
TABLE 2

Results of trials of multiphase screening for colorectal cancer (CRC) with fecal occult blood testing as the first stage

\begin{tabular}{|c|c|c|c|c|}
\hline Study & Mandel et al (21-24) & Kronborg et al (25) & Hardcastle et al $(26,27)$ & Kewenter et al $(28,29$ \\
\hline Level of evidence* & $\mathrm{I}$ & $\mathrm{I}$ & 1 & $\mathrm{I}$ \\
\hline Age of subjects (years) & 50 to 80 & 45 to 74 & 50 to 74 & 60 to 64 \\
\hline Number of subjects in the screened group & 31,157 & 30,967 & 76,466 & 31,144 \\
\hline Number of subjects in the control group & 15,394 & 30,966 & 76,384 & 34,164 \\
\hline Compliant patients (\%) & $75-78$ & 67 & 59 & 66 \\
\hline \multirow[t]{2}{*}{ Test sensitivity (\%) } & 49.5 (annual) & 48 & 48 & 79 \\
\hline & 38.3 (biennial) & & & \\
\hline Positive predictive value (\%) & 2.2 & 10.2 to 17.0 & 9.9 to 11.9 & 4.6 to 5.0 \\
\hline Follow-up (years) & 13 & 10 & Median 7.8 & Median 8.3 \\
\hline \multirow[t]{3}{*}{ CRC deaths at last follow-up, $n$ (rate/10,000) } & Annual $82(53)$ & Screen $360(47)$ & Screen 121 (35) & Screen 205 (66) \\
\hline & Biennial $117(75)$ & Control 420 (55) & Control $138(40)$ & Control $249(80)$ \\
\hline & Control $121(79)$ & & & \\
\hline \multirow{3}{*}{$\begin{array}{l}\text { Relative risk of CRC death with } \\
\text { screening }(95 \% \mathrm{Cl})\end{array}$} & Annual 0.67 (0.51 to 0.89 ) & $0.86(0.74$ to 0.99$)$ & $0.85(0.74$ to 0.98$)$ & $0.82(0.68$ to 0.99$)$ \\
\hline & Biennial 0.95 (0.74 to 1.23$)$ & & & \\
\hline & Total 0.81 (0.65 to 1.02$)$ & & & \\
\hline
\end{tabular}

Note that the test performance characteristics are reported per patient and not per test. *See Appendix 1 (Table 3) for classification of level of evidence (17). Table modified from reference 12

Clinical efficacy of screening tests

Screening with the FOBT: Four large randomized controlled trials (RCTs) (21-29) assessed the Hemoccult test (Beckman Coulter, USA) in screening individuals at average risk and the results of these were summarized in an excellent review (12) (Table 2) (Level I).

In the University of Minnesota Colon Cancer Control Study (Level I), the death rate from CRC was lower in the group in which patients had been screened annually (21-24). In an updated report (22) (18 years follow-up) the significant reduction in mortality was also seen in the group screened biennially. The cumulative rate of deaths averted over 13 years of follow-up was 2.95 per 1000 for subjects screened annually, and 0.5 deaths averted per 1000 subjects screened biennially. The rate of positive FOBT slides (rehydrated) was 9.8\% among 5917 subjects screened annually and 4365 subjects screened biennially for a detection rate of 646 cases of cancer. This study also reported decreases in the incidence of CRC of 0.83 (95\% CI: 0.70 to 0.90$)$ and 0.80 (95\% CI 0.73 to 0.94$)$ in the annu$\mathrm{al}$ and biennial screening groups respectively (21-24).

In a Danish study (Level I), tumours were discovered at earlier stages in the screened group; significantly more subjects in the screened group, compared to in the control group, had local $(10 \%$ versus $5 \%, \mathrm{P}<0.01)$ and curative surgery $(69 \%$ versus $59 \%, \mathrm{P}<0.01)$ for their cancers $(25,30)$. Total mortality did not differ between groups, but the overall relative risk (RR) for CRC deaths was 0.82 (95\% CI: 0.68 to 0.99).

In a United Kingdom study (Level I), individuals were randomized by household and the compliance rate was only $59.6 \%(26,27)$. No dietary restrictions were required before initial testing, but the Hemoccult test was repeated on a special diet if positive. The overall RR for CRC deaths was 0.85 (95\% CI: 0.74 to 0.98 ). Only $4 \%$ of all patients underwent colonoscopic examinations during the study. Almost one-half of all cancers in the screened group were detected in the noncompliant subjects. In this study, only $25 \%$ of all cancers in the screened group were detected by the Hemoccult testing; also, $74 \%$ of all screen-detected cancers were noted in the rectum or sigmoid. In addition, colonic adenomas were found in 1001 subjects in the screened group (267 over $2 \mathrm{~cm}$ in size) compared with 370 subjects in the control group (100 over $2 \mathrm{~cm}$ ).

The final study was conducted in Sweden (Level I) $(28,29)$. Patients were tested after a special diet and retested if positive before proctoscopy, flexible sigmoidoscopy and barium enema. Further tests were performed in $3.8 \%$ of subjects after the first screen, and $5.1 \%$ after the second. The overall risk reduction to date is $12 \%$, which is not statistically significant (12).

A meta-analysis including all four trials and two nonrandomized cohort studies, for a total of over 443,000 patients, demonstrated a $16 \%$ risk reduction in CRC-related mortality ( $\mathrm{RR}=0.84,95 \%$ CI 0.77 to 0.93) (31) (Level I). This effect increased to $23 \%$ in compliant patients ( $R R=0.77,95 \%$ CI 0.57 to 0.89 ). If 10,000 subjects were offered screening, 8.5 (95\% CI 3.6 to 13.5 ) deaths from CRC would be prevented over 10 years. The number needed to screen over 10 years to prevent one death is 1173 .

Summary of the effectiveness of the FOBT test, and outstanding issues: Screening with the Hemoccult test results in a significant decrease in CRC-related mortality, demonstrating a RR reduction of approximately 15\% (Level I). In absolute terms, approximately $8.5 \mathrm{CRC}$ deaths would be averted if 10,000 subjects were screened over 10 years.

The RR reduction in mortality rates is fairly constant, but the detection rate of CRC is higher in older individuals, suggesting that a higher proportion of lives saved relative to the number screened would be achieved if screening is started later on in life.

The modest sensitivity of the FOBT (approximately 50\%) has caused some authors to question the attributable effect of mortality reduction and to suggest that chance selection for colonoscopy, which was performed in a large proportion of subjects, may have accounted for between one-third and one-half of the observed benefit (32). They have questioned the need 
for FOBT, and whether colonoscopic screening alone should be carried out. None of these studies assessed the psychological issues of screening, its acceptability, or the costs of such a program. Such considerations are critical because participation rates in other studies have not been as high as the $60 \%$ seen in the United Kingdom study (26,33).

Screening with sigmoidoscopy: The Kaiser Multiphasic Health Checkup Evaluation Study was a RCT, in which subjects aged 35 years to 54 years were actively encouraged (study group) or not encouraged (control group) to have a sigmoidoscopy as part of a multiphase health check-up (34) (Level I). The authors then conducted a retrospective chart review comparing patients who had died from CRC with matched control subjects (Level II-2). Of the patients who died from CRC, $8.8 \%$ had undergone sigmoidoscopy within the preceding 10 years, compared with $24.2 \%$ of the control subjects. The adjusted odds ratio (OR) was 0.41 (95\% CI 0.25 to 0.69). A similar analysis of patients with fatal colon cancer beyond the reach of the sigmoidoscope compared with a control group found no differences (35) (Level II-2). These results suggest that patients with fatal rectal cancer were less likely than matched controls to have undergone rigid sigmoidoscopy in the preceding 10 years.

A similar trial reported by Newcomb et al (36) included 74 case subjects and 206 control subjects (Level II-2). There were no differences in rates of FOBT; however, 30\% of control versus $10.6 \%$ of case subjects had a sigmoidoscopy $(\mathrm{OR}=0.21,95 \% \mathrm{CI} 0.08$ to 0.52$)$. The reduction was observed in cases in which cancer was limited to the rectum and distal colon only.

A third case-control study included 30,000 individuals followed in the Veteran Affairs system in the United States, including 8722 patients who had died of colon cancer and 7629 of rectal cancer $(37,38)$ (Level II-2). The odds of a patient dying of a colon or a rectal cancer having had an endoscopic procedure (flexible sigmoidoscopy, colonoscopy, polypectomy) were 0.51 (95\% CI 0.44 to 0.58 ) and 0.55 (95\% CI 0.47 to 0.64 ), respectively, compared with control subjects.

Two large descriptive studies with adequate follow-up (Level II-1) demonstrated that screening with sigmoidoscopy can decrease the mortality rate from CRC compared with that seen in the normal population (39-41). However, these are lower quality studies than the FOBT trials and a number of biases cannot be ruled out due to the study methodology.

Verne et al (42) conducted a small RCT comparing FOBT with flexible sigmoidoscopy or combined FOBT and flexible sigmoidoscopy. The trial included 3744 patients aged 50 years to 75 years randomly assigned by household (Level I). Compliance was greater in the group assigned to flexible sigmoidoscopy (46.6\%) alone versus FOBT (31.6\%)or both $(30.1 \%)$. Polyps were noted in $17 \%$ of patients, with adenomas in $6.8 \%$ and high-risk adenomas in $2.4 \%$. Cancers were found in $0.4 \%$ of patients. The addition of FOBT did not improve the detection rates. Based on histology, $7 \%$ of subjects required follow-up colonoscopy. Mortality rates were not reported.

Another RCT reported by Berry et al (43) assigned 6371 patients aged 50 years to 74 years to FOBT alone or FOBT and flexible sigmoidoscopy (Level I). Compliance rates were $48 \%$ and $50 \%$ for FOBT in the two groups, respectively, but only $20 \%$ agreed to go on to a flexible sigmoidoscopy. Despite this, the cancer detection rate was fourfold higher in the FOBT/flexible sigmoidoscopy group compared with the
FOBT alone group (8.9 per 1000 screened versus 2.0/1000 screened), with most of the difference related to the addition of flexible sigmoidoscopy. In a third trial, Rasmussen et al (44) randomly assigned 10,978 subjects aged 50 years to 75 years to undergo FOBT alone or FOBT and flexible sigmoidoscopy (Level I). Compliance was lower with the combined procedure than in FOBT alone (40\% versus 50\%), but more lesions were detected (CRC: 12 versus four, and adenomas: 72 versus 14).

Recent prevalence studies have brought into question the sensitivity of a screening program based on FOBT and/or flexible sigmoidoscopy. Imperiale et al (45) demonstrated that approximately one-half of the cases of advanced proximal neoplasias would be missed if colonoscopic screening was performed only in persons with distal polyps (Level II-1). Lieberman et al (46) determined the prevalence of neoplasia and the sensitivity of one-time screening with FOBT (rehydrated Hemoccult II) plus sigmoidoscopy in 2885 asymptomatic patients aged 50 years to 75 years at low risk for CRC (Level II-1). Using colonoscopy as the reference test, they identified all patients with advanced neoplasias (adenoma $1 \mathrm{~cm}$ or more, a villous adenoma or an adenoma with highgrade dysplasia or invasive cancer). Of these, $23.9 \%$ had a positive FOBT for a RR of advanced neoplasia of 3.47 (95\% CI 2.76 to 4.35 ) compared with subjects who had a negative FOBT. Sigmoidoscopy identified $70.3 \%$ of all subjects with advanced neoplasia; the addition of FOBT did not increase the detection rates significantly $(75.8 \%)$. Thus, one-time screening with both FOBT and flexible sigmoidoscopy would fail to detect $24 \%$ of subjects with advanced neoplasia. Moreover, FOBT was not helpful in diagnosing small tubular adenomas.

The United Kingdom flexible sigmoidoscopy screening trial (47) assessing the usefulness of a single flexible sigmoidoscopy screening offered at age 60 years is underway, with results not yet available.

Summary of the trials examining the role of sigmoidoscopy: There is evidence from three case-control studies suggesting that sigmoidoscopy may reduce the rate of CRC mortality (Level II-2). In addition, three RCTs suggest that flexible sigmoidoscopy may be superior to FOBT in detecting adenomas and possibly cancer (Level I). However, these studies are small and do not report mortality data. For technical reasons, it is reasonable to assume that flexible sigmoidoscopy may be preferable to rigid sigmoidoscopy, and that the flexible instrument may be more acceptable to patients. One-time screening with both FOBT and flexible sigmoidoscopy would fail to detect $24 \%$ of subjects with advanced neoplasia (46) (Level II-1).

Controversies remain around the issues of frequency, compliance and feasibility of sigmoidoscopy; the need for colonoscopic follow-up examinations; and the significance of small polyps. Two ongoing RCTs may be helpful in elucidating such issues (18).

Screening with colonoscopy: No direct evidence exists concerning the role of screening with colonoscopy. However, in the University of Minnesota trial, 38\% of individuals underwent colonoscopy, which resulted in a $\mathrm{RR}$ reduction in CRC mortality of $33 \%$ ( $R R=0.67,95 \%$ CI 0.50 to 0.87 ) compared with control subjects (21-24) (Level II-1). The reanalysis of the data by Lang and Ransohoff (32) suggested that some of the reduction in mortality was attributable to chance selection of individuals for colonoscopy (Level III). Less frequent screening with colonoscopy might, therefore, be more appropriate than 
initial yearly or biennial screening with FOBT testing. A comparison of the results of the National Polyp Study (an RCT designed to determine the optimal frequency of colonoscopy in individuals who underwent polypectomy) (48) with historical controls from three groups of patients (St-Mark's hospital, the Mayo Clinic and a general population registry (the Surveillance, Epidemiology and End Results [SEER]) provides some evidence that colonoscopy may decrease the risk of cancer, but provides no evidence that it reduces mortality (Level II-3) (48).

Four uncontrolled trials examining asymptomatic, low-risk patients over the age of 50 years showed that the incidence of adenomas (0.22) and CRC (0.01) were higher with colonoscopy than with FOBT, and compliance rates were variable (6\% to $49 \%)$ (49-51) (Level III).

Screening with barium enema: DCBE has not been evaluated in clinical trials as a screening test for CRC. A well-structured screening program is needed to better define the role of this test in screening for CRC and its impact on survival $(1,52)$ (Level II-3).

\section{Cost effectiveness of screening tests}

Screening policies are costly and therefore cost-effectiveness analyses have been done, most of which have used modelling approaches (see reviews by Provenzale [53], Crott [54] and Pignone et al [55]). Results of cost-effectiveness modelling analyses suggest that all screening programs are associated with a cost and none appear to be cost saving; however, the same is true for breast and cervical cancer screening programs (56).

A cost-effectiveness analysis was done based on two RCTs (United Kingdom [27] and Denmark [25,30]), in which FOBT was offered biannually from the age of 50 years on (57). The estimates of cost per quality-adjusted life-years (QALYs) gained showed that CRC screening was similar in terms of cost effectiveness to breast cancer screening in the short term, and superior in the long term. The incremental cost effectiveness ratio of the most efficient program compared favourably to other screening programs in Denmark (58).

A modelling analysis that accounted for compliance rates compared a strategy of a 10-year repeat colonoscopy as the primary screening tool, annual FOBT and sigmoidoscopies every five years with positive tests followed by colonoscopy (59). The sigmoidoscopy was the most costly strategy, where the cost is dominated by colonoscopy. Conversely, Khandker et al (60) showed that five-yearly sigmoidoscopy was the preferred method, followed by annual FOBT, with DCBE and colonoscopy screening being very similar to each other. Glick et al (61) found that a sigmoidoscopy-based screening approach was most effective with intermediate values for DCBE and DCBE with FOBT. Here too, colonoscopy screening was not cost-effective due to its high cost. Using more recent estimates and standardized methodologies, MacMahon et al (62) suggested that strategies with FOBT alone were less cost-effective and colonoscopy-based strategies were favoured. Annual FOBT with three-yearly DCBE was very costly, but flexible sigmoidoscopy-based alternatives were not analyzed. An Italian model developed by Sorrentino et al (63) showed increasing costs per death prevented for policies of single colonoscopy at age 50 years, 10-yearly colonoscopy, five-yearly sigmoidoscopy, annual FOBT and FOBT with five-yearly sigmoidoscopy (63).
Two studies examined the use of a one-time screening colonoscopy at different ages (between 45 years and 60 years) $(64,65)$. The effectiveness of screening as measured in QALYs was highest at age 50 years, but the costs were lowest at age 60 years. In both, screening was more cost-effective than not screening, and screening below age 50 years was prohibitively costly.

The net cost per life-year-saved with a once-only FOBT and flexible sigmoidoscopy for individuals aged 60 years found that screening for the early detection and prevention of CRC is one of the most cost-effective programs in cancer (66). Similarly, a single screen for CRC with either sigmoidoscopy or colonoscopy appeared to be a highly cost-effective intervention when compared with breast screening using mammography. The sigmoidoscopy approach cost less but had a lower yield than colonoscopy (67). An analysis based on the American Gastroenterological Association recommendations found that annual FOBT with sigmoidoscopy every five years was the most cost-effective, and overall, the cost-effectiveness ratios compared favourably with those of cervical screening in the United States (68). Screening with colonoscopy was very sensitive to its cost and to compliance assumptions.

A recently published systematic review on cost-effectiveness analyses of CRC screening concluded that such a program appears cost-effective compared with no screening, but that a single optimal strategy cannot be determined from the currently available data (55).

Summary of cost-effectiveness data: Although no cost savings can be expected from introducing a program of CRC screening, the cost-effectiveness of such an implementation would appear favourable when compared with other screening programs, including breast or cervical cancer. Studies suggest that compliance with FOBT may be low in everyday practice, and its high false-positive rate makes this option less costeffective.

A once-only screening strategy may be attractive owing to its low cost, but also is dependent on the polyp detection rate with regard to the number of follow-up examinations to be performed. Depending on the transition probability of polyps larger than $1 \mathrm{~cm}$, and assuming that only these need removal, DCBE could be valuable but is dependent on its test performance in an everyday-practice setting. The respective roles of colonoscopy and sigmoidoscopy depend largely on the relative costs of one versus the other, and may favour the adoption of colonoscopy in Canada, and especially Quebec, in contrast to the United States. The compliance with colonoscopy and the availability of resources must also be considered.

\section{Summary of background data}

Screening with the FOBT: There is Level I evidence from RCTs that screening with FOBT reduces mortality in asymptomatic patients with no risk factors who are over age 50 years. The RR of CRC death is 0.84 (95\% CI 0.77 to 0.93 ) overall, and 0.77 (95\% CI 0.57 to 0.89 ) in compliant individuals. There remain concerns about the high rate of false positive results, feasibility and small clinical benefits of such screening (over 1000 subjects must be screened for 10 years to avert one death from CRC).

Screening with barium enema: The reported performance characteristics of radiological tests vary, but would favour the use of the DCBE technique. No outcomes studies exist to allow a grading of existing evidence, yet comparative effectiveness 
with regard to detection is about $80 \%$ to $85 \%$. It is commonly used and is included in the recommendations of many groups. Screening with sigmoidoscopy: There is evidence from case control studies (Level II-2) that flexible sigmoidoscopy reduces mortality from $\mathrm{CRC}$ in patients over the age of 50 years. However, the evidence regarding whether it should be performed alone or with FOBT is unclear. Recent data suggest that one-time screening with both FOBT and flexible sigmoidoscopy would fail to detect $24 \%$ of subjects with advanced neoplasia (Level II-1).

Screening with colonoscopy: Evidence remains unclear with respect to the use of colonoscopy as an initial screening test. Of all the screening tests, it exhibits the highest diagnostic yield in terms of the detection of adenomas and carcinomas (Level II-3). It also allows synchronous polypectomy, but may not be feasible for screening asymptomatic patients because of patient compliance, the expertise and equipment required, and the associated costs. Perhaps a less frequent screening interval may obviate some of these concerns.

Recommendations by other professional organizations A summary of some recent recommendations, which were considered in the development of these guidelines, is included in Appendix 2 (Table 4) (1,15,16,69-74). The updated recommendations from the United States Multisociety Task Force on Colorectal Cancer (74) were published after the Association des gastro-entérologues du Québec (AGEQ) Task Force had completed its work, but have been included as a reference.

\section{FEASIBILITY OF A SCREENING PROGRAM IN QUEBEC}

The feasibility of a screening policy for CRC in Quebec was evaluated based on the number of barium enemas and colonoscopies performed in Quebec over a one-year period from April 1, 2000 through March 30, 2001 and on the population of Quebec aged 50 years to 79 years (Appendix 3). Excluding any follow-up therapeutic or surveillance procedures, a screening program of 10-yearly colonoscopy (for $50 \%$ of the target population) and five-yearly DCBE (for the other $50 \%$ of the target population) would require 121,500 procedures per year. A program of 10-yearly colonoscopy (for $50 \%$ of the target population) and 10 -yearly DCBE (for the other $50 \%$ of the target population) would require 81,000 procedures per year. It is clear from these estimates that a combination of screening modalities (colonoscopy and DCBE) is required, considering available human and technological resources.

\section{CONCLUSIONS}

CRC, a leading cause of death, is a curable cancer that is preventable with appropriate screening. The AGEQ Task Force evaluated FOBT, endoscopy (including sigmoidoscopy and colonoscopy), barium enema and virtual colonoscopy. Although most clinical efficacy data are available for FOBT and sigmoidoscopy, these strategies are limited by the high false positive rate, low compliance and low detection yield of FOBT. In fact, even a combination of these strategies will miss $24 \%$ of cancers. Colonoscopy is the best strategy to both detect and remove polyps and to diagnose CRC. DCBE is also a sensitive detection method but controlled trials of its use as a screening tool are needed.

The AGEQ Task Force recommended the establishment, in Quebec, of a screening program with 10-yearly colonoscopy or five- to 10-yearly DCBE for individuals aged 50 years or more at low risk. Although any screening program is associated with a cost, evidence suggests that screening for CRC can reduce mortality rates, with cost-effectiveness similar to that of initiatives for breast and cervical cancer.

This position paper was written at the request of the AGEQ with recognized participation from representatives from the Association des Radiologues du Québec and the Association des Chirurgiens Généraux du Québec.

ACKNOWLEDGEMENTS: The Committee would like to thank the assistance of Ms Sylvie Bergeron and Ms Pauline Lavigne in preparing this manuscript.

\section{APPENDIX 1}

\section{GRADING FOR LEVELS OF EVIDENCE AND RECOMMENDATIONS}

\section{TABLE 3}

Categorization of evidence and recommendations (17)

\section{Quality of evidence}

I Evidence obtained from at least one properly randomized controlled trial.

II-1 Evidence obtained from well-designed controlled trials without randomization.

II-2 Evidence obtained from well-designed cohort or case-control analytical studies, preferably from more than one centre or research group.

II-3 Evidence obtained from comparisons between times or places with or without the intervention, or dramatic results in uncontrolled experiments.

III Opinions of respected authorities, based on clinical expertise, descriptive studies or reports of expert committees.

Classification of recommendations

A There is good evidence to support the procedure or treatment.

B There is fair evidence to support the procedure or treatment.

C There is poor evidence to support the procedure or treatment, but recommendations may be made on other grounds.

D There is fair evidence that the procedure or treatment should not be used.

E There is good evidence that the procedure or treatment should not be used. 


\section{APPENDIX 2}

RECOMMENDATIONS OF VARIOUS ORGANIZATIONS

TABLE 4

Guidelines for tests and screening intervals in persons at average risk for colorectal cancer* from major American societies (18)

\begin{tabular}{|c|c|c|c|c|}
\hline Test & $\begin{array}{l}\text { United States Preventive } \\
\text { Services Task Force (69) }\end{array}$ & $\begin{array}{l}\text { Gastrointestinal } \\
\text { Consortium (1) }\end{array}$ & $\begin{array}{l}\text { American College of } \\
\text { Gastroenterology (70) }\end{array}$ & $\begin{array}{c}\text { American } \\
\text { Cancer Society (71) }\end{array}$ \\
\hline Year of recommendation & 1996 & 1997 & 2000 & 2001 \\
\hline Frequency of three-sample FOBT alone & Yearly & Yearly & Yearly & Yearly \\
\hline Frequency of sigmoidoscopy alone & Unspecified & Every five years & Every five years & Every five years \\
\hline \multicolumn{5}{|l|}{ Frequency of three-sample } \\
\hline FOBT alone + sigmoidoscopy & Insufficient evidence & Yearly and every five years & Yearly and every five years & Yearly and every five years \\
\hline Frequency of colonoscopy & Insufficient evidence & Every 10 years & Every 10 years (preferred) & Every 10 years \\
\hline Frequency of barium enema & Insufficient evidence & Every five to 10 years & Every five to 10 years & Every five years \\
\hline
\end{tabular}

${ }^{*}$ Screening for colorectal cancer in these subjects should start at age 50 years. FOBT Fecal occult blood testing

Guidelines for screening for colorectal cancer in persons at normal risk from the Canadian Task Force on Preventive Health (2001) (16)

1. There is good evidence to support the inclusion of annual or biennial fecal occult blood testing (FOBT) (A recommendation).

2. There is fair evidence to include a flexible sigmoidoscopy in the periodic health examinations of asymptomatic individuals over age 50 years (B recommendation).

3. There is insufficient evidence to make recommendations about whether only one or both of FOBT and sigmoidoscopy should be performed ( $\mathrm{C}$ recommendation).

4. There is insufficient evidence to include or exclude colonoscopy as an initial screen in the periodic health examination ( $\mathrm{C}$ recommendation).

Guidelines for screening for colorectal cancer from the Conseil d'évaluation des technologies de la santé du Québec (2000) (15) "In conclusion, the Conseil believes that a colorectal cancer (CRC) screening program would lead to significant reduction in the mortality due to this type of cancer. In light of the existing evidence, such a program would target asymptomatic persons aged 50 to 79 years and would involve biennial screening by FOBT. A single positive finding would result in an invitation to an interview and a medical examination followed by a diagnostic and, if need be, therapeutic colonoscopy. The choice of a primary screening modality is still preliminary, since current research is exploring the direct use of colonoscopy, sigmoidoscopy or double-contrast barium enema (DCBE).

"However, there should be various prerequisites both for instituting the proposed program and exploring other screening strategies, including setting up feasibility studies and pilot trials for the purpose of validating the operational parameters in Québec. Also, conducting such studies will require additional research support."

Guidelines for screening for CRC from the Ontario Expert Panel of Cancer Care Ontario (1999) (73)

The following are recommendations from the Expert Panel on CRC Screening to the Board of Cancer Care Ontario. They represent the consensus view of the Expert Panel.

The Expert Panel took into account the following considerations in determining its recommendations:
1. Evidence of efficacy and effectiveness from the scientific medical literature,

2. Explorations from the evidence on one screening test to another screening test, based on the observed characteristics of both tests,

3. Acceptability of the proposed test to the Ontario public health care providers, and

4. Capacity of the Ontario health care system to provide the screening test in the context of a provincial program.

The Expert Panel focused on a broad policy of issues. It has produced policy recommendations and not clinical guidelines. The Expert Panel recognizes that there are many critical technical and operational issues that still must be addressed.

1. Cancer Care Ontario should develop and introduce a program of CRC screening for average-risk individuals. This program should have the following features:

i. The program should be available to all individuals aged 50 years or older. There should be active recruitment of individuals aged 50 to 75 years old.

ii. The program should use FOBT as its primary modality. The program should be based on a protocol that produces parameters of sensitivity, specificity and predictive value similar to, or better than, the results of published randomized control trials.

iii. Individuals with an abnormal test result with FOBT (ie, a positive test on any sample) should be offered an accurate examination of the entire colon and rectum. This can be either by colonoscopy or DCBE with flexible sigmoidoscopy. The choice of assessment modality should be based on accessibility and the preference of the patient and their health care provider. In assessments with DCBE, it may be possible to limit the use of flexible sigmoidoscopy to a subset of patients with sigmoid colons that are difficult to visualize radiologically. This issue should be evaluated by the program and any appropriate protocol changes introduced.

iv. The program should be expanded to include the option of direct visualization of the entire colon (ie, 
colonoscopy or DCBE) as a primary modality. This expansion should, however, only be contemplated when the program is assured that there is sufficient colonoscopy and DCBE capacity to provide timely assessment of FOBT-positive individuals, appropriate screening of high-risk individuals and diagnostic assessment of symptomatic individuals. In the meantime, the program should consider evaluative studies of colonoscopy and DCBE screening to assess issues like screening frequency, compliance, provider acceptance and cost.

v. The program should assess the benefits of combining screening with flexible sigmoidoscopy and FOBT, by the development and assessment of evaluative studies.

2. Cancer Care Ontario should establish a representative multistakeholder advisory structure to provide ongoing direction regarding the design and operation of the $\mathrm{CRC}$ screening program. This advisor structure should have the capacity to provide advice on policy, technical issues, evaluation and research. Membership should include strong consumer representation as well as representation from health care providers, academics, ethicists and privacy experts.

3. Cancer Care Ontario should consider implementing a program for CRC screening if the following conditions are met:

- Screening must be comprehensive, including patient recruitment, follow-up and recall and timely assessment.

- Public and provider education must be a priority. Public education must address the cultural and linguistic diversity of Ontario's population and the unique needs of hard-to-reach groups.

- All people in Ontario much have reasonable access to screening.

- Individuals participating in screening should only do so with informed consent, based on understanding of the possible harms and realistic benefits of screening. Participation should be voluntary, with the ability to opt-out at any time.

- The screening program, in all of its aspects, must be of high quality, supported by minimum standards, evidencebased guidelines and promotion of best practice.

- All aspects of screening must be subject to continuous monitoring and evaluation with the capacity to change standards, guidelines and best practices based on the evaluation.

- There must be commitment to modify screening standards, guidelines and best practices based on new scientific evidence.

- The program must be supported by an effective and efficient information system designed to accommodate the needs for confidentiality and information sharing.

- There must be provision of adequate resources (financial, physical and human) to support all aspects of screening, including assessment.

- Screening should, as much as possible, be accommodated within the existing patterns of practice and referral of the Ontario health care system.
Guidelines for screening for CRC in persons at normal risk from the United States Preventive Services Task Force (2002) (72)

The United States Preventitive Services Task Force (USPSTF) found fair to good evidence that several screening methods are effective in reducing mortality from CRC. The USPSTF concluded that the benefits from screening substantially outweigh potential harms, but the quality of evidence, magnitude of benefit and potential harms vary with each method.

The USPSTF found good evidence that periodic FOBT reduces mortality from $\mathrm{CRC}$ and fair evidence that sigmoidoscopy alone or in combination with FOBT reduces mortality. The USPSTF did not find direct evidence that screening colonoscopy is effective in reducing CRC mortality rates; efficacy of colonoscopy is supported by its integral role in trials of FOBT, extrapolation from sigmoidoscopy studies, limited case-control evidence and the ability of colonoscopy to inspect the proximal colon. DCBE offers an alternative means of whole-bowel exam, but is less sensitive than colonoscopy and there is no direct evidence that it is effective in reducing mortality rates. The USPSTF found insufficient evidence that newer screening techniques (for example, computed tomographic colography) are effective in improving health outcomes.

There are insufficient data to determine which screening strategy is best in terms of the balance of benefits and potential harms or cost-effectiveness. Studies reviewed by the USPSTF indicate that CRC screening is likely to be cost-effective (less than $\$ 30,000$ per additional year of life gained) regardless of the strategy chosen.

It is unclear whether the increased accuracy of colonoscopy compared with alternative screening methods (for example, the identification of lesions that FOBT and flexible sigmoidoscopy would not detect) offsets the procedure's additional complications, inconvenience and costs.

Guidelines for screening for CRC in persons at average risk from the United States Multisociety Task Force on CRC (2003) (74)

Men and women at average risk should be offered screening with one of the following options beginning at age 50 years. The rationale for presenting multiple options is that no single test is of unequivocal superiourity and that giving patients a choice allows them to apply personal preferences and may increase the likelihood that screening will occur. The strategies are not equal with regard to evidence of effectiveness, magnitude of effectiveness, risk or up-front costs. Reviewing the rationale section for each screening test (presented in the original guideline document) will provide clinicians with information that they can use in presenting the relative effectiveness of each test to patients.

FOBT: Offer yearly screening with FOBT using a guaiac-based test with dietary restriction or an immunochemical test without dietary restriction. Two samples from each of three consecutive stools should be examined without rehydration. Patients with a positive test on any specimen should be followed up with colonoscopy.

Sigmoidoscopy: Offer flexible sigmoidoscopy every five years.

Combined FOBT and flexible sigmoidoscopy: Offer screening with FOBT every year combined with flexible sigmoidoscopy every five years. When both tests are performed, the FOBT should be done first.

Colonoscopy: Offer colonoscopy every 10 years.

DCBE: Offer DCBE every five years. 


\section{APPENDIX 3}

\section{ESTIMATING THE DEMAND FOR SCREENING PROCEDURES FOR COLORECTAL CANCER IN QUEBEC}

Although some screening is currently carried out, it is impossible to precisely estimate the extent, as no dedicated billing codes exist for this procedure (regardless of how it is performed).

Quebec statistics that are relevant to this discussion are included in Tables 5 and 6.

\section{Assumptions}

\section{RESULTS OF A FEASIBILITY ESTIMATE}

- Quebecois aged 50 to 79 years: 2,000,000

- Assuming the 10-yearly colonoscopy/double contrast barium enema (DCBE) screening program, the demand would be 200,000 per year CSCOPE.

- Remove those who are too ill (10\%) and those already having been screened (approximately 25\% in the United States).

- Also remove the noncompliant patients (assume compliance of $60 \%$ based on mammography).

Findings

- If 10-yearly colonoscopy (for 50\% of the target population) and five-yearly DCBE (for the other 50\% of the target population) were carried out, 121,500 procedures would need to be done yearly.

- If 10 -yearly colonoscopy (for 50\% of the target population) and 10-yearly DCBE (for the other $50 \%$ of the target population) were carried out, 81,000 procedures would need to be done yearly.

- Note that these estimates exclude any follow-up therapeutic or surveillance procedures.

\section{REFERENCES}

1. Winawer SJ, Fletcher RH, Miller L, et al. Colorectal cancer screening: Clinical guidelines and rationale. Gastroenterology 1997;112:594-642.

2. National Cancer Institute of Canada. Canadian Cancer Statistics, 2000. Toronto: National Cancer Institute of Canada, 2000.

3. Tierney RP, Ballantyne GH, Modlin IM. The adenoma to carcinoma sequence. Surg Gynecol Obstet 1990;171:81-94.

4. Morson BC, Dawson IMP. Gastrointestinal Pathology. Oxford: Blackwell Scientific, 1972

5. Greene FL. Distribution of colorectal neoplasms. A left to right shift of polyps and cancer. Am Surg 1983;49:62-5.

6. Helwig EB. Ademnomas and the pathogenesis of cancer of the colon and rectum. Dis Colon Rectum 1959;2:5.

7. Prager ED, Swinton NW, Young JL, Veidenheimer MC, Corman ML. Follow-up study of patients with benign mucosal polyps discovered by proctosigmoidoscopy. Dis Colon Rectum 1974;17:322-4.

8. Muto T, Bussey HJ, Morson BC. The evolution of cancer of the colon and rectum. Cancer 1975;36:2251-70.

9. Morson BC. The evolution of colorectal carcinoma. Clin Radiol 1984;35:425-31.

10. Moore J, Cowled P. Hereditary non-polyposis colorectal cancer syndrome. Aust N Z J Surg 1999;69:6-13.

11. Vasen HF, Griffioen G, Tops CM, et al. Familial adenomatous polyposis and its clinical surveillance. Neth J Med 1993;42:105-8.

12. McLeod RS. Screening strategies for colorectal cancer: A systematic review of the evidence. Can J Gastroenterol 2001;15:647-60.
TABLE 5

Frequency of single contrast (SC) and double contrast (DC) barium enema in a medium-sized hospital in Quebec from April 1, 2000 to March 30, 2001

\begin{tabular}{lcc}
\hline Patient age (years) & $\begin{array}{c}\text { Frequency } \\
\text { (SC) }\end{array}$ & $\begin{array}{c}\text { Frequency } \\
\text { (DC) }\end{array}$ \\
\hline$<50$ & 4826 & 9654 \\
$50-59$ & 3001 & 6917 \\
$60-69$ & 3174 & 6075 \\
$70-79$ & 2803 & 3996 \\
$\geq 80$ & 877 & 929 \\
Not available & 13,788 & 37,102 \\
Total (in a medium-sized hospital) & 28,459 & 64,673 \\
\hline
\end{tabular}

TABLE 6

Frequency of colonscopy (Procedure code 697) in a medium-sized Quebec hospital from April 1, 2000 to March 30, 2001

\begin{tabular}{lc}
\hline Patient age (years) & Frequency \\
\hline$<50$ & 11,909 \\
$50-59$ & 10,569 \\
$60-69$ & 10,056 \\
$70-79$ & 8186 \\
$\geq 80$ & 2596 \\
Total & 43,316 \\
\hline
\end{tabular}

- Moreover, in Quebec, 43,316 colonoscopies were performed between 2000 and 2001 (29,000 amongst patients aged 50 to 80 years) and 93,000 barium enemas were completed (37,000 double contrast), with 30,000 (21,000 double contrast) in 50 to 80 year olds.

It is thus clear that a combination of screening modalities (colonoscopy and DCBE) is required, considering available human and technological resources.

13. Imperiale TF, Wagner DR, Lin CY, et al. Results of screening colonoscopy among persons 40 to 49 years of age. N Engl J Med 2002;346:1781-5.

14. Benson IA. Recent and ongoing clinical trials for treating colorectal cancer. Exp Opin Invest Drugs 2002;11:871-80.

15. Battista RN. Conseil d'évaluation des technologies de la santé du Québec. Le dépistage du cancer colorectal. (CETS 99-2RF) Montreal: CETS, 1999:xx-146.

16. Canadian Task Force on Preventive Health Care. Colorectal cancer screening. Recommendation statement from the Canadian Task Force on Preventive Health Care. CMAJ 2001;24;165:206-8.

17. Goldbloom R, Battista RN. The periodic health examination: 1. Introduction. CMAJ 1986;134:721-3.

18. Ransohoff DF, Sandler RS. Clinical practice. Screening for colorectal cancer. N Engl J Med 2002;346:40-4.

19. Gazelle GS, McMahon PM, Scholz FJ. Screening for colorectal cancer. Radiology 2000;215:327-35.

20. Rex DK. Barium studies/virtual colonoscopy: The gastroenterologist's perspective. Gastrointest Endosc 2002;55(Suppl 7):S6,S33-6.

21. Mandel JS, Bond JH, Church TR, et al. Reducing mortality from colorectal cancer by screening for fecal occult blood. Minnesota Colon Cancer Control Study. N Engl J Med 1993;328:1365-71.

22. Mandel JS, Church TR, Ederer F, Bond JH. Colorectal cancer mortality: Effectiveness of biennial screening for fecal occult blood. J Natl Cancer Inst 1999;91:434-7. 
23. Mandel JS, Church TR, Bond JH, et al. The effect of fecal occultblood screening on the incidence of colorectal cancer. N Engl J Med 2000;343:1603-7.

24. Mandel JS, Bond JH, Snover DC, et al. The University of Minnesota's Colon Cancer Control Study: Design and progress to date. In: Chamberlain J, Miller AB, eds. Screening for Gastrointestinal Cancer. Toronto: Huber, 1998:434-7.

25. Kronborg O, Fenger C, Olsen J, Jorgensen OD, Sondergaard O. Randomised study of screening for colorectal cancer with faecaloccult-blood test. Lancet 1996;348:1467-71.

26. Hardcastle JD, Thomas WM, Chamberlain J, et al. Randomised, controlled trial of faecal occult blood screening for colorectal cancer. Results for first 107,349 subjects. Lancet 1989;1:1160-4.

27. Hardcastle JD, Chamberlain JO, Robinson $\mathrm{MH}$, et al. Randomised controlled trial of faecal-occult-blood screening for colorectal cancer. Lancet 1996;348:1472-7.

28. Kewenter J, Brevinge H, Engaras B, Haglind E, Ahren C. Results of screening, rescreening, and follow-up in a prospective randomized study for detection of colorectal cancer by fecal occult blood testing. Results for 68,308 subjects. Scand J Gastroenterol 1994;29:468-73.

29. Kewenter J, Bjork S, Haglind E, et al. Screening and rescreening for colorectal cancer. A controlled trial of fecal occult blood testing in 27,700 subjects. Cancer 1988;62:645-51.

30. Kronborg O, Fenger C, Olsen J, Bech K, Sondergaard O. Repeated screening for colorectal cancer with fecal occult blood test. A prospective randomized study at Funen, Denmark. Scand J Gastroenterol 1989;24:599-606.

31. Towler B, Irwig L, Glasziou P, et al. A systematic review of the effects of screening for colorectal cancer using the faecal occult blood test, hemoccult. BMJ 1998;317:559-65.

32. Lang CA, Ransohoff DF. Fecal occult blood screening for colorectal cancer. Is mortality reduced by chance selection for screening colonoscopy? JAMA 1994:271:1011-3.

33. Blalock SJ, DeVellis BM, Sandler RS. Participation in fecal occult blood screening: A critical review. Prev Med 1987;16:9-18.

34. Friedman GD, Collen MF, Fireman BH. Multiphasic Health Checkup Evaluation: A 16-year follow-up. J Chronic Dis 1986;39:453-63.

35. Selby JV, Friedman GD, Quesenberry CP Jr, Weiss NS. A casecontrol study of screening sigmoidoscopy and mortality from colorectal cancer. N Engl J Med 1992;326:653-7.

36. Newcomb PA, Norfleet RG, Storer BE, Surawicz TS, Marcus PM. Screening sigmoidoscopy and colorectal cancer mortality. J Natl Cancer Inst 1992;84:1572-5.

37. Muller AD, Sonnenberg A. Prevention of colorectal cancer by flexible endoscopy and polypectomy. A case-control study of 32,702 veterans. Ann Intern Med 1995;123:904-10.

38. Muller AD, Sonnenberg A. Protection by endoscopy against death from colorectal cancer. A case-control study among veterans. Arch Intern Med 1995;155:1741-8.

39. Gilbertsen VA. Proctosigmoidoscopy and polypectomy in reducing the incidence of rectal cancer. Cancer 1974;34(Suppl):936-9.

40. Gilbertsen VA, Nelms JM. The prevention of invasive cancer of the rectum. Cancer 1978;41:1137-9.

41. Hertz R, Deddish M, Day E. Value of periodic examinations in detecting cancer of the colon and rectum. Postgrad Med $1960 ; 27: 290-4$.

42. Verne JE, Aubrey R, Love SB, Talbot IC, Northover JM. Population based randomized study of uptake and yield of screening by flexible sigmoidoscopy compared with screening by faecal occult blood testing. BMJ 1998;317:182-5.

43. Berry DP, Clarke P, Hardcastle JD, Vellacott KD. Randomized trial of the addition of flexible sigmoidoscopy to faecal occult blood testing for colorectal neoplasia population screening. Br J Surg 1997;84:1274-6.

44. Rasmussen M, Kronborg O, Fenger C, Jorgensen OD. Possible advantages and drawbacks of adding flexible sigmoidoscopy to hemoccult-II in screening for colorectal cancer. A randomized study. Scand J Gastroenterol 1999;34:73-8.

45. Imperiale TF, Wagner DR, Lin CY, et al. Risk of advanced proximal neoplasms in asymptomatic adults according to the distal colorectal findings. N Engl J Med 2000;343:169-74.

46. Lieberman DA, Weiss DG. One-time screening for colorectal cancer with combined fecal occult-blood testing and examination of the distal colon. N Engl J Med 2001;345:555-60.

47. Atkin WS, Edwards R, Wardle J, et al. Design of a multicentre randomised trial to evaluate flexible sigmoidoscopy in colorectal cancer screening. J Med Screen 2001;8:137-44.

48. Winawer SJ, Zauber AG, Ho MN, et al. Prevention of colorectal cancer by colonoscopic polypectomy. The National Polyp Study Workgroup. N Engl J Med 1993;329:1977-81.
49. Lieberman D. ASGE Clinical Update: Colon Cancer Screening: Role of endoscopy. ASGE 2000;8:1-4.

50. Rex DK, Sledge GW, Harper PA, et al. Colonic adenomas in asymptomatic women with a history of breast cancer. Am J Gastroenterol 1993;88:2009-14.

51. Johnson DA, Gurney MS, Volpe RJ, et al. A prospective study of the prevalence of colonic neoplasms in asymptomatic patients with an age-related risk. Am J Gastroenterol 1990;85:969-74.

52. Gazelle GS, McMahon PM, Scholz FJ. Screening for colorectal cancer. Radiology 2000;215:327-35.

53. Provenzale D. Cost-effectiveness of screening the average-risk population for colorectal cancer. Gastrointest Endosc Clin N Am 2002;12:93-109.

54. Crott R. The cost-effectiveness of screening for colorectal cancer. Exp Rev Pharmacoeconomics Outcomes Res 2001;1:89-98.

55. Pignone M, Saha S, Hoerger T, Mandelblatt J. Cost-effectiveness analyses of colorectal cancer screening: A systematic review for the US Preventive Services Task Force. Ann Intern Med 2002;137:96-104.

56. Loeve F, Brown ML, Boer R, et al. Endoscopic colorectal cancer screening: A cost-saving analysis. J Natl Cancer Inst 2000;92:557-63.

57. Whynes DK, Neilson AR, Walker AR, Hardcastle JD. Faecal occult blood screening for colorectal cancer: Is it cost-effective? Health Econ 1998;7:21-9.

58. Gyrd-Hansen D. Fecal occult blood tests. A cost-effectiveness analysis. Int J Technol Assess Health Care 1998;14:290-301.

59. Sonnenberg A, Delco F, Inadomi JM. Cost-effectiveness of colonoscopy in screening for colorectal cancer. Ann Intern Med 2000;133:573-84.

60. Khandker RK, Dulski JD, Kilpatrick JB, et al. A decision model and cost-effectiveness analysis of colorectal cancer screening and surveillance guidelines for average-risk adults. Int J Technol Assess Health Care 2000;16:799-810.

61. Glick S, Wagner JL, Johnson CD. Cost-effectiveness of doublecontrast barium enema in screening for colorectal cancer. AJR Am J Roentgenol 1998;170:629-36.

62. McMahon PM, Bosch JL, Gleason S, et al. Cost-effectiveness of colorectal cancer screening. Radiology 2001;219:44-50.

63. Sorrentino D, Paduano R, Bernardis V, Piccolo A, Bartoli E. Colorectal cancer screening in Italy: Feasibility and cost- effectiveness in a model area. Eur J Gastroenterol Hepatol 1999;11:655-60.

64. Ness RM, Holmes AM, Klein R, Dittus R. Cost-utility of one-time colonoscopic screening for colorectal cancer at various ages. Am J Gastroenterol 2000;95:1800-11.

65. Ransohoff DF, Lang CA. Cost effectiveness of one-time colonoscopy screening to reduce colorectal cancer mortality. Gastroenterology 1994;106:A24.

66. Norum J. Prevention of colorectal cancer: A cost-effectiveness approach to a screening model employing sigmoidoscopy. Ann Oncol 1998;9:613-8.

67. Geul KW, Bosman FT, van Blankenstein M, Grobbee DE, Wilson JH. Prevention of colorectal cancer. Costs and effectiveness of sigmoidoscopy. Scand J Gastroenterol Suppl 1997;223:79-87.

68. Frazier AL, Colditz GA, Fuchs CS, Kuntz KM. Cost-effectiveness of screening for colorectal cancer in the general population. JAMA 2000;284:1954-61.

69. US Preventative Services Task Force USPSTF. Screening for Colorectal Cancer. Guide to Clinical Preventative Services. Alexandria: International Medical Publishing Inc, 1996

70. Rex DK, Johnson DA, Lieberman DA, Burt RW, Sonnenberg A. Colorectal cancer prevention 2000: Screening recommendations of the American College of Gastroenterology. Am J Gastroenterol 2000;95:868-77.

71. Smith RA, von Eschenbach AC, Wender R, et al. American Cancer Society guidelines for the early detection of cancer: Update of early detection guidelines for prostate, colorectal, and endometrial cancers. Also: Update 2001 - Testing for early lung cancer detection. CA Cancer J Clin 2001;51:38-75,7-80.

72. US Preventative Service Task Force USPSTF. Screening for colorectal cancer: Recommendation and rationale. Ann Intern Med 2002;137:129-31.

73. Cancer Care Ontario. Ontario expert panel on colorectal cancer. Final report of the Ontario expert panel on colorectal cancer, 1999. $<$ http://www.cancercare.on.ca/pdf/colorectal.pdf $>$ (Version current at July 19, 2004)

74. Winawer S, Fletcher R, Rex D, et al. Colorectal cancer screening and surveillance: Clinical guidelines and rationale - Update based on new evidence. Gastroenterology 2003;124:544-60. 


\section{ERRATUM}

AN Barkun, $G$ Jobin, $G$ Cousineau, et al. The Quebec Association of Gastroenterology position paper on colorectal cancer screening - 2003. Can J Gastroenterol 2004;18(8):509-519.

The original translation of this abstract contained an error. The abstract and the corrected translation are printed below. Our apologies are extended to the authors.

Colorectal cancer is a leading cause of death and the third most common cancer in Canada. Evidence suggests that screening can reduce mortality rates and the cost effectiveness of a program compares favourably with initiatives for breast and cervical cancer.

The objectives of the Association des gastro-entérologues du Québec Task Force were to determine the need for a policy on screening for colorectal cancer in Quebec, to evaluate the testing methods available and to propose one or more of these alternatives as part of a formal screening program, if indicated.

Fecal occult blood testing (FOBT), endoscopy (including sigmoidoscopy and colonoscopy), barium enema and virtual colonoscopy were considered. Although most clinical efficacy data are available for FOBT and sigmoidoscopy, there are limitations to programs based on these strategies. FOBT has a high false positive rate and a low detection yield, and even a combination of these strategies will miss $24 \%$ of cancers. Colonoscopy is the best strategy to both detect and remove polyps and to diagnose colorectal cancer, with double contrast barium enema also being a sensitive detection method.

The Task Force recommended the establishment, in Quebec, of a screening program with five- to 10-yearly double contrast barium enema or 10-yearly colonoscopy for individuals aged 50 years or older at low risk. The program should include outcome monitoring, public and professional education to increase awareness and promote compliance, and central coordination with other provincial programs. The program should be evaluated; specific billing codes for screening for colorectal cancer would help facilitate this. Formal feasibility, effectiveness and cost-effectiveness studies in Quebec are now warranted.

Le document de principes de l'Association des gastro-entérologues du Québec sur le dépistage du cancer colorectal 2003

Le cancer colorectal est une cause importante de décès et le troisième cancer en importance au Canada. Selon des données probantes, le dépistage peut réduire les taux de mortalité, et le rapport coût-efficacité d'un programme se compare favorablement avec les initiatives relatives au cancer du sein et du col de l'utérus.

Les objectifs du groupe de travail de l'Association des gastro-entérologues du Québec consistaient à déterminer le besoin d'une politique de dépistage du cancer colorectal au Québec, à évaluer les méthodes d'essai disponibles et à proposer une ou plusieurs de ces possibilités dans le cadre d'un programme de dépistage officiel, s'il y a lieu.

Le test de sang occulte fécal (TSOF) et l'endoscopie (y compris une sigmoïdoscopie et une coloscopie), le lavement baryté et la coloscopie virtuelle ont été envisagés. Bien que la plupart des données d'efficacité clinique soient disponibles pour le TSOF, la sigmoïdoscopie, les programmes fondés sur ces stratégies comportent des limites. Le TSOF s'associe à un taux faux positif élevé et à un faible rendement de détection, et même une combinaison de ces stratégies passera à côté de $24 \%$ des cancers. Toutefois, la coloscopie représente la meilleure stratégie à la fois pour déceler et extraire les polypes et pour diagnostiquer le cancer colorectal, et le lavement baryté en double contraste constitue une méthode de détection sensible.

Le groupe de travail a recommandé la mise en œuvre, au Québec, d'un programme de dépistage comportant un lavement baryté en double contraste aux cinq à dix ans ou une coloscopie aux dix ans pour les personnes de 50 ans ou plus à faible risque. Ce programme devrait inclure l'évaluation des issues, l'éducation publique et la formation professionnelle pour accroitre la sensibilisation et promouvoir le respect du traitement et une coordination centralisée avec d'autres programmes provinciaux. Le programme devrait être évalué. Des codes de facturation précis pour le dépistage du cancer colorectal faciliteraient cette évaluation. Maintenant, des études officielles de faisabilité, d'efficacité et de rapport coût-efficacité au Québec s’imposent. 


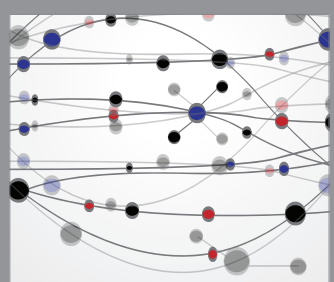

The Scientific World Journal
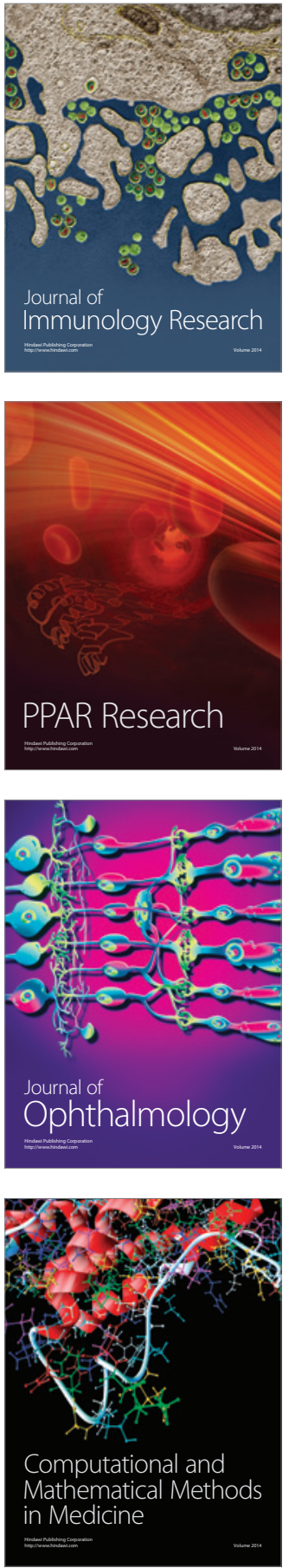

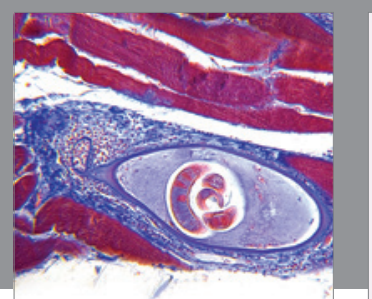

Gastroenterology Research and Practice

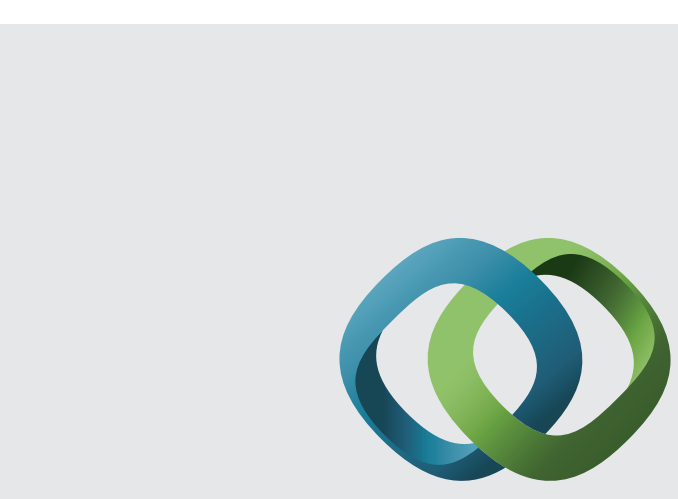

\section{Hindawi}

Submit your manuscripts at

http://www.hindawi.com
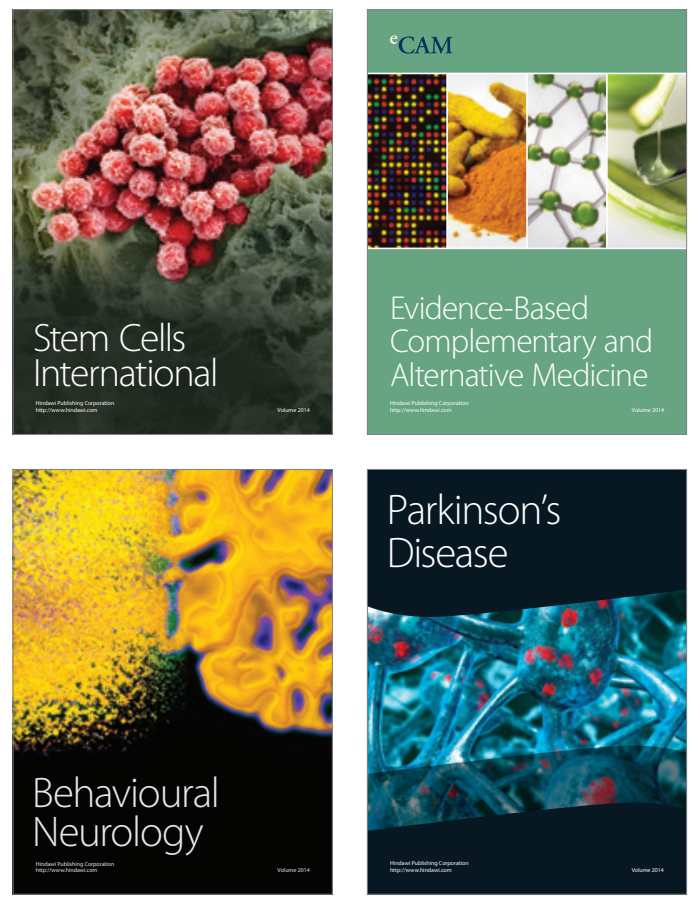
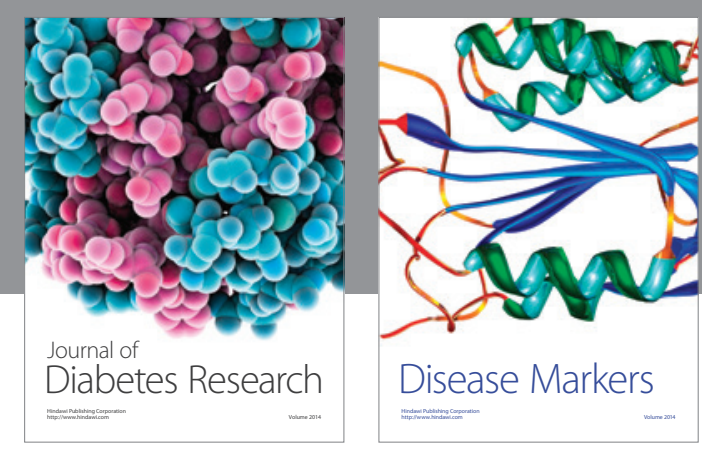

Disease Markers
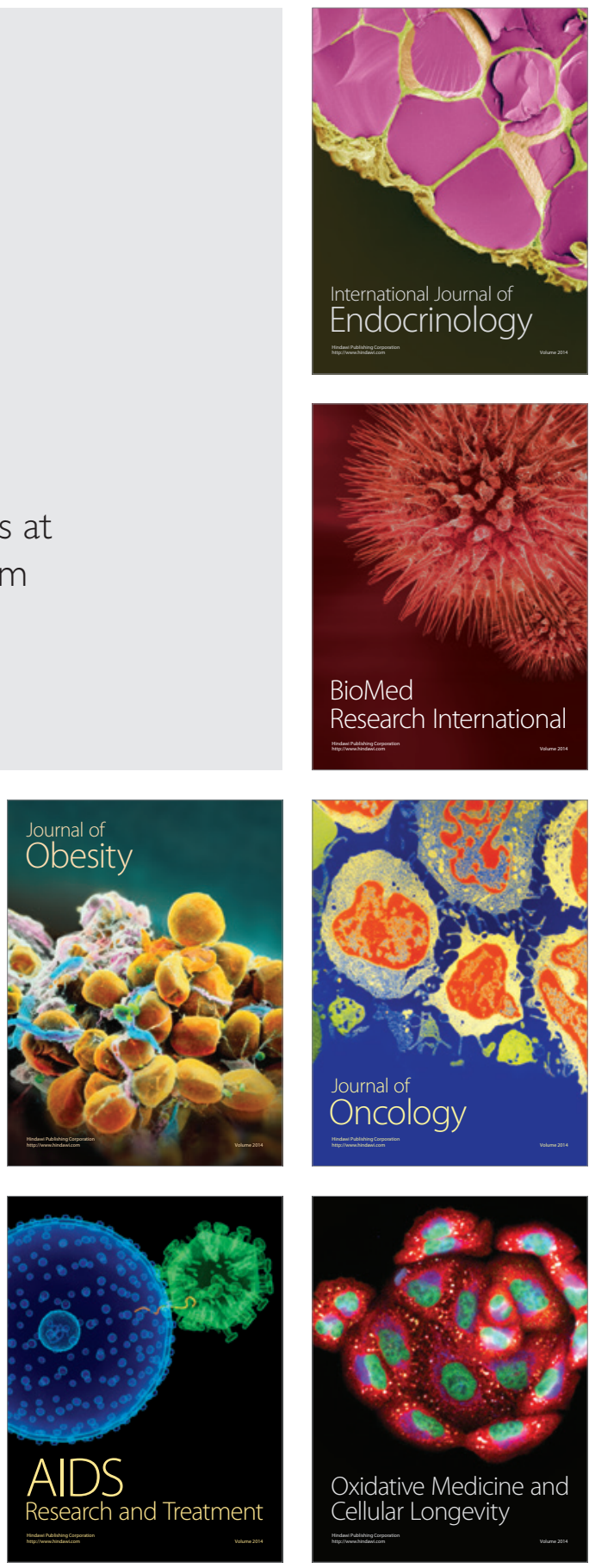